\title{
Prevention and Care of Physical Injuries when Playing Table Tennis at Home during COVID-19 Quarantine
}

ISSN: 2577-1914

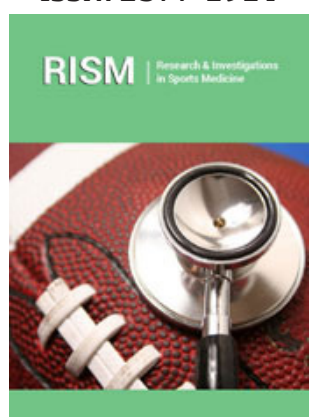

*Corresponding author: Yi Zhang, Department of Physical Education, Shanghai University, Shanghai 20444, China

Submission: 㘹 April 28, 2020

Published: 侮 May 29, 2020

Volume 6 - Issue 3

How to cite this article: Yi Zhang, Haodong Gu. Prevention and Care of Physical Injuries when Playing Table Tennis at Home during COVID-19 Quarantine. Res Inves Sports Med, 6(3): RISM.000639. 2020. DOI: $10.31031 /$ RISM.2020.06.000639

Copyright@ Yi Zhang, This article is distributed under the terms of the Creative Commons Attribution 4.0 International License, which permits unrestricted use and redistribution provided that the original author and source are credited.

\author{
Yi Zhang* and Haodong Gu \\ Department of Physical Education, Shanghai University, China
}

\begin{abstract}
Table tennis is a sport suitable for different types of environmental conditions and can be carried out both indoor and outdoor with simple equipment. Due to the convenience and flexibility of this sport, it has become a globally favorite exercise for people who are confined at home due to COVID-19 outbreak. Unlike common misperceptions about table tennis being a light exercise, it is a fast sport with quick physical movement, posture changes, and even body rotations, which can easily get players injured. Particularly, people are prone to injuries in a home environment that often does not fully meet the standard of playing conditions. Common injuries caused by playing this sport are around the wrist, waist, knee, and shoulder areas. In this article, illustrations and discussed are centered on injury prevention, analysis, and therapeutic care. Cautious preparations in advance, both physically and environmentally, can effectively prevent much of the injuries.
\end{abstract}

\section{Introduction}

Table tennis is one of the most popular sports in the world that is adopted by the Olympic Games. Today table tennis is one of the most popular participant sports in the world. There are currently 226 national membership associations within the International Table Tennis Federation (ITTF). According to the ITTF (2020), a total of 1.41 billion viewers watched key ITTF events in the 2019 season, including the World Table Tennis Championships, the World Cup and the World Tour (not including the year-end final in December, as final figures are still being confirmed). In addition, ITTF-owned social media has grown exponentially, growing by over 1 million fans in a single calendar year: up from 2,802,725 in 2018 to 3,845,493 in 2019, marking a 37\% increase (excluding digital TV and online streaming subscribers). The total number of video viewership of table tennis on the four major social platforms (YouTube, Facebook, Instagram and Twitter) grew from 155 million to 350 million during the 2019 season. Logically, the boosted spectatorship and media viewership would attract more participants to play table tennis.

Over the years, table tennis has gained popularity in China and become a widely respected and popular sport. A single board or concrete slab with a row of bricks in the middle can serve as a table tennis table, which does not take up much space and is ideal for densely populated China. Most importantly, table tennis is a sport for people of both genders, all ages, and different physical conditions, and also for all walks of life. To take part in this sport, people only need a pair of rackets, a ball, a table, and a net. People can be even creative in changing the size of equipment to play the game at home or in other locations with limited space. As a popular leisure activity, many schools, factories, office buildings, and households in China are equipped with table tennis tables [1].

In 2020, COVID-19 outbreak became global. In order to prevent the contagious virus from spreading, people have responded to the government calls for social distancing and staying home, which brought some new challenges and problems to people's health, such as weight gain, loss of appetite, gastrointestinal dysfunction, poor sleep, and mood anxiety. Indoor physical exercises can be one of the effective solutions to these problems. Physical 
benefits of participating in sport activities are well documented, which include both health-related and motor-related fitness $[2,3]$. According to Wade [4], regular exercises may reinforce and improve one's immune system, which is particularly relevantin this pandemic. Regular participation in exercise enhances bone density, tendon and ligament intensity, range of motion, and lean body mass (muscular strength). The physical effects of exercise also include increase in cardiorespiratory efficiency, decrease in body fat, and protection against chronic diseases [5]. Improvements in psychological well-being have also been observed as a result of physical activities. Proper exercise and sport activities lead to a reduction in mental status of negative valence such as anxiety, depression, anger, and loneliness [4]. Nevertheless, caused by the virus pandemic, public places such as sport venues, fitness clubs, and parks and trails are shut down for an extended period of time. Home-based exercise became a new fitness choice for people to maintain health conditions, improve immunity, and relieve negative emotions such as anxiety and depression.

Table tennis is a sport that is suitable for different types of environmental conditions and can be carried out both indoor and outdoor with simple equipment. Due to the convenience and flexibility of this sport, table tennis has become a globally favorite exercise for people who are confined at home. The ball of table tennis is small and fast moving; when playing this sport, the cerebral cortex of the player is in an excited condition all the time with restrained alternant process, which exerts a very good exercising effect to the acuity that improves vision and the flexibility of nervous system. Because the ball moves fast and spins, the players are required to accurately observe the rotation and landing point of the ball instantly and quickly decide a strategy to return the ball play. This process helps improving the function of the nervous system and the coordination of the body $[1,6,7]$. Based on these advantages of table tennis, during COVID-19 many people choose to work out by playing table tennis in their living rooms or basements, and normally they get a good work-out from doing so.

Table tennis may be considered by many as a light exercise and players are less prone to injuries. Yet, in reality, this is not true. Table tennis is a fast sport with quick physical movement, posture changes, and even body rotations, which requires full attention and swift reaction [8,9]. The fast-paced game and regularly-rotating body movements can easily get players injured. Particularly, people are prone to injuries in a home environment that often does not fully meet the standard of playing conditions. Unchecked environmental conditions can be major concerns to the safety and wellbeing of individuals choosing to play table tennis at home. Challenges may include, but are not limited to the following: (a) a family's living room or basement may not be spacious enough to allow for a full range of motion and free body movement, (b) the floor and light conditions may not meet the minimum requirements of playing table tennis, (c) people may not wear proper sport shoes, and (d) people do not do adequate warm-up exercises, such as stretching, before playing the game. Common injuries caused by playing table tennis are around the wrist, waist, knee, and shoulder areas. Careful preparations in advance, both physically and environmentally, can effectively prevent much of the injuries.

\section{Wrist Injury and Prevention Measures}

The wrist is mainly made up of eight small bones of different shapes, which is why the wrist can make rather flexible and accurate movements. In the meantime, small bones in the wrist are susceptible to injuries. The range of motion of the wrist is overall small. When players hitting the ball, their arms act as a lever and the wrist makes the inward rotation or abduction motion quickly. If the wrist joint is lack of flexibility, it is prone to an injury or even repeated injures. There are three major measures to prevent a wrist injury: (a) warm up, stretch, and get the wrist ready for playing, (b) form a good habit of using protectors such as wrist band, and (c) avoid over exertion of strength and full range of motion. When the wrist is slightly injured, symptoms such as pain, limited movement, and swelling can appear. While ice pad helps reducing inflammation in early hours, fumigation with medicine and appropriate massage would promote blood stasis absorption after 48 72 hours of injury. As massage is an adjunctive therapy, the following specific methods of massage can be applied in a wrist injury: (a) wrist flexor: when the wrist is in dorsiflexion, massaging from the inside of the forearm can stretch the flexor of the wrist so as to accurately massage the muscle group. To do so, one can use the forearm and fist to effectively replace the thumb when massaging; and (b) wrist extensor: these muscles are usually more fibrotic than the flexors and an accurate massage of each muscle will yield a good effect when wrist flexion will put the extensor into a stretching state [9-11].

\section{Waist Injury and Prevention Measures}

When playing table tennis, the upper body of the player leans forward and the lumbar spine ligament is pressed forward also. For an extended period of time, the sacrospinous muscle is in the state of contraction and tension. Many players are not aware of the necessity of relaxing and stretching after ending the exercise, which often leads to muscles over-fatigue. Accumulation of fatigues lead to damage to local muscle groups. The waist movement is mainly controlled by large muscle groups. In the game, almost every stroke connects to the motion of rotating the waist. If there is not enough warm-up, or the player lacks muscle group strength, endurance, and preparedness, the inertia of the large muscle group cannot be easily overcome when one tries to rotate the waist quickly, which may cause the waist injury easily. To prevent an injury, people playing the game need to adopt full warm-up of the waist, the process of which usually takes 2 5 minutes. When a slight injury of local soft tissue or ligament occurs, one needs to reduce or stop the activity immediately, and relax the muscle group through appropriate 
massage, cupping, plaster, or other therapeutic procedures. Massage can be an adjunctive therapy. The following is a specific method of massage for waist injury: Massage the quadratus psoas by using the fingers or knuckles, which allows more precise and deeper access to the quadratus psoas. When there is a major lumbar soft tissue or ligament tear, obviously facet joint dislocation, or lumbar disc herniation, the injured individual needs to rest in bed and seek for medical treatment $[8,10,12]$.

\section{Knee Joint Injury and Prevention}

During the course of playing table tennis, the knee joint is always in a semi-flexion position and the ligaments around the joint are under strenuous tension. In a sudden overdraft, it is easy to cause damage to the collateral ligaments of both sides of the knee joint. At time, the knee joint injury is also related to the players' playing style. For the choppers, the lower limbs have a large range of motion and a fast speed, resulting in the heavy burden on the knee joint, exposing the knees to injuries in this way. Therefore, people can adjust the style of playing according to the individual situation, strengthening the knee joint by resistance and flexibility training to reduce the occurrence of injuries, such as strengthening the power of quadriceps through the standing exercising and squatting stances, and improving the flexibility of the hamstrings by leg pressing and splitting. In order to prevent injuries, some precautions should be taken while playing. Similar to the measures to prevent any injuries, the priority is to warm up sufficiently before playing, including jogging, joint rotation, and stretching. Secondly, individuals should not only practice forehand or backhand, which is unbalanced and prone to muscle strain. Generally speaking, if an individual intends to play for more than an hour, it is better to limit the consecutive playing time to half an hour. Players can walk for a while during the intervals to relax their muscles and eliminate fatigue. Massage can be an adjunctive therapy. Here is a method of sitting position to relax knee joint: sit next to the massage table, fix the sore spot or use one's fingers to restrict movement, and then stretch the knee joint. In more severe cases, full extension should be stopped to reduce the likelihood of inflammation, which can develop as a result of pressure on the patella $[8,9,12]$.

\section{Shoulder Joint Injury and Prevention}

When playing table tennis, an individual develops the tendency of using the arm to smash the ball repeatedly. The repetitive movements involving over-using or over-exerting the shoulder and excessively pulling shoulder may easily cause an injury to the tendon in the long head of biceps brachia. The main reasons of injuries are that the shoulder joint is pulled repeatedly and rub with ligament when doing the activities of extension and rotation. In order to prevent injuries, some precautions should be taken while playing. First, it is important to warm up sufficiently before playing, including jogging, joint rotation, and stretching. Secondly, one should not only practice forehand or backhand strokes, which is unbalanced and prone to muscle strain. In addition, it is better for people to play for half an hour maximum in each round. Players should take a break afterwards, for instance taking a walk during the intervals to relax their muscles and eliminate fatigue. When feeling uncomfortable or slightly injured, one can self-massage shoulder blades on the back with fingers. These can be performed by using fingers or knuckles for physical therapy and manipulating arms to stretch the muscles on one's side. If the area is too tense or sensitive, the player can lower the arm to soften the muscle and make the area easier to access $[8,9,10,11]$.

\section{Discussion and Summary}

Table tennis is a fun, recreational and easily accessible participation sport. Its simple rules of playing, low requirement on equipment, and abundant flexibility for adaptation perfectly fit the need for indoor exercise during the COVID-19 pandemic. Even when a playing partner is not available, an individual can still play the game against a wall that bounces the ball back to the player. For convenience and better game experience, small tables and colored balls can be used at homes. Adjustable can also be its playing pace and intensity, making table tennis suitable for people with different background, physical condition, and skill levels. The well-documented physical, mental, psychological, and even social benefits of playing table tennis $[9,10,12]$ indicate that it can be a good choice of sport activity for individuals, family members, and friends in social distancing time.

To achieve the benefits of playing table tennis, preventing injuries and reduce the severity of injuries are important. Doing so relies on an adequate preparation in terms of home environment, warm-up exercises, assessment of one's fitness conditions, and selfcontrol for the intensity of playing. Injuries should be highly wary of with precaution and prevention measures being applied before, during, and after the play. While physical injuries are harmful, unpleasant, and undesirable under general circumstance, injuries during COVID-19 would be worse as many medical facilities and services are not fully open to regular patients. Thus, preventing injuries, major injures in particular, is of priority in the play. As most households have limited space, individuals need to make efforts to select or adjust the size of the table, managing the environment to avoid tripping, running into objects, or even hitting a wall. Players should also check the floor condition to avoid slipping and switch on adequate lights for better playing experience. One can also install good ventilations to avoid over-heating or excessive low temperature in the playing room [13-17].

Good physical warm-ups can be achieved through walking in the house, stretching various muscle groups and joints, and gradually increasing the intensity of the play. Elevation of playing pace and intensity should take individual background, physical conditions, and skill levels into considerations. Oftentimes, a moderate level of intensity together with a moderate amount of exercise would fit the 
condition of home exercise. Various forms of health-related fitness exercises would help an individual with good physical condition to get prepared for the game. Taking advantage of whatever equipment, devices, and objects are available in a home, people can do isometric and isotonic muscular strength or endurance training, aerobic dance, stationary bicycle riding, static walking or jogging, and other types of stretching and movement aimed to maintain and improve flexibility, agility, balance, and stability. Assuming that the Internet is available, it would be in an individual's best interest to seek information on injury prevention and care in case of light injuries. Learning care procedures and particularly massage protocols for body segments that are prone to injuries add to the safety level of playing table tennis at home, especially when medical services are inconvenient or even unavailable due to the global fight of COVID-19. Nonetheless, in the event of a major injury, an individual should seek diagnosis and treatment from well-trained medical care professionals.

\section{References}

1. Zhang Y, Kim M, Wang JJ, Pitts BG (2018) Reversing the tide of sport globalization from west to east? Examining consumer demand for table tennis clubs in the U.S. International Journal of Sports Marketing and Sponsorship 19(2): 217-235.

2. Baumgartner TA, Jackson AS, Mahar MT, Rowe DA (2016) Measurement for evaluation in kinesiology. ( $9^{\text {th }}$ edn), Jones \& Bartlett, Burlington, MA, USA.

3. Zhou L, Wang JJ, Chen X, Cianfrone B, Pifer ND (2019) Community-sport service provision, participant satisfaction, and participation: Experience perspective of Guangdong, China. International Journal of Sports Marketing and Sponsorship 21(1): 127-147.
4. Wade P (2020) Exercise may boost your immune system. Kinesiology Today 13(2): 7-8.

5. Blair SN, Clark DG, Cureton KJ, Powell KE (1989) Exercise and fitness in childhood: Implications for a lifetime of health. In: Gisolfi CV, Lamb DR (Eds.), Perspectives in exercise science and sports medicine: Youth exercise and sport. Benchmark, Indianapolis, USA, pp. 401-422.

6. Allen JB (1996) Table tennis and motor development. International Journal of Table Tennis Sciences 3: 131-134.

7. SharpBrains (2014) Trend: playing table tennis to enhance brain fitness and mental health.

8. Lin XY (1998) Introduction to table tennis. ( $2^{\text {nd }}$ edn), Anhui Science \& Technology, Hefei, China.

9. Xiao DD (2019) Biomechanical study of table tennis technology. Chinese Books, Beijing, China.

10. Lu AF (2017) A guide to physical training for table tennis players. Tongji University, Shanghai, China.

11.Su PR (1995) Teaching and training of table tennis. People's Sports, Beijing, China.

12. Tang JJ (2003) Table tennis skills: Technical and tactical diagram. Beijing Sport University, Beijing, China.

13. International Table Tennis Federation (2020) A history of table tennis in the olympic games.

14. International Table Tennis Federation (2020) Directory.

15. International Table Tennis Federation (2020) History of table tennis.

16. International Table Tennis Federation (2020) Table Tennis sets new digital \& broadcast media records in 2019.

17. Letts G (2018) A brief history of table tennis (Ping-Pong). 